\title{
DBU-Mediated cleavage of aryl- and heteroaryl disulfides
}

\author{
Dubekile Nyoni, ${ }^{a}$ Kevin A. Lobb, ${ }^{a}$ Perry T. Kaye ${ }^{\text {a*and }}$ Mino R. Caira ${ }^{b}$ \\ ${ }^{a}$ Department of Chemistry, Rhodes University, Grahamstown, South Africa \\ ${ }^{b}$ Department of Chemistry, University of Cape Town, Rondebosch, South Africa \\ E-mail:P.Kaye@ru.ac.za
}

\begin{abstract}
The capacity of the nitrogen nucleophile, 1,8-diazabicyclo[5.4.0]undec-7-ene (DBU) to reduce aryl- and heteroaryl disulfides to the corresponding mercaptans is demonstrated. While dicarboxylated disulfide analogues afford the mono-DBU disulfide salts, as confirmed by X-ray crystallography, the corresponding methyl esters are cleaved normally.
\end{abstract}

Keywords: Disulfide cleavage, aryl disulfides, DBU, aryl mercaptans

\section{Introduction}

The synthesis of $2 \mathrm{H}$-1-benzothiopyrans (thiochromenes) has typically involved the condensation of thiophenols with acrylic acid derivatives, followed by reduction and dehydration, ${ }^{1}$ and a number of approaches to these systems have been reported. ${ }^{2-4}$ As part of our ongoing research on applications of Baylis-Hillman methodology, ${ }^{5}$ we reported the convenient, one-step synthesis of the thiochromenes 3a-g via the 1,8-diazabicyclo[5.4.0]undec-7-ene (DBU)-catalyzed reaction of 2,2'-dithiodibenzaldehyde 1a with activated alkenes 2a-g (Scheme 1). ${ }^{6}$ The thiochromenes 3ag were obtained in a single step - an observation that suggested the capacity of DBU to reduce the disufides 4a-g (formed via the Baylis Hillman reaction), possibly via the sequence outlined in Scheme $1 .{ }^{6}$ Phosphine nucleophiles have been implicated in the direct cleavage of disulfides, ${ }^{7}$ while photo-induced cleavage via disulfide radical anions has been attributed to electron transfer from excited-state aniline ${ }^{8}$ and various amines have been used in large excess (40 eq.) to produce, in situ at elevated temperatures, benzenethiyl radicals from diphenyl disulfide via a single electron transfer process. ${ }^{9}$ DBU has been used as a base in thiazolium salt-catalyzed disufide reduction-aldehyde oxidation processes ${ }^{10}$ but, to our knowledge, its role as a nucleophile in the direct cleavage of disulfides is unprecedented. We now report the results of further research directed at exploring the general capacity of DBU to reduce diaryl and heterodiaryl disulfides to mercaptans in the absence of an activated alkene, thus precluding involvement of a Baylis-Hillman adduct, as suggested in Scheme 1. 


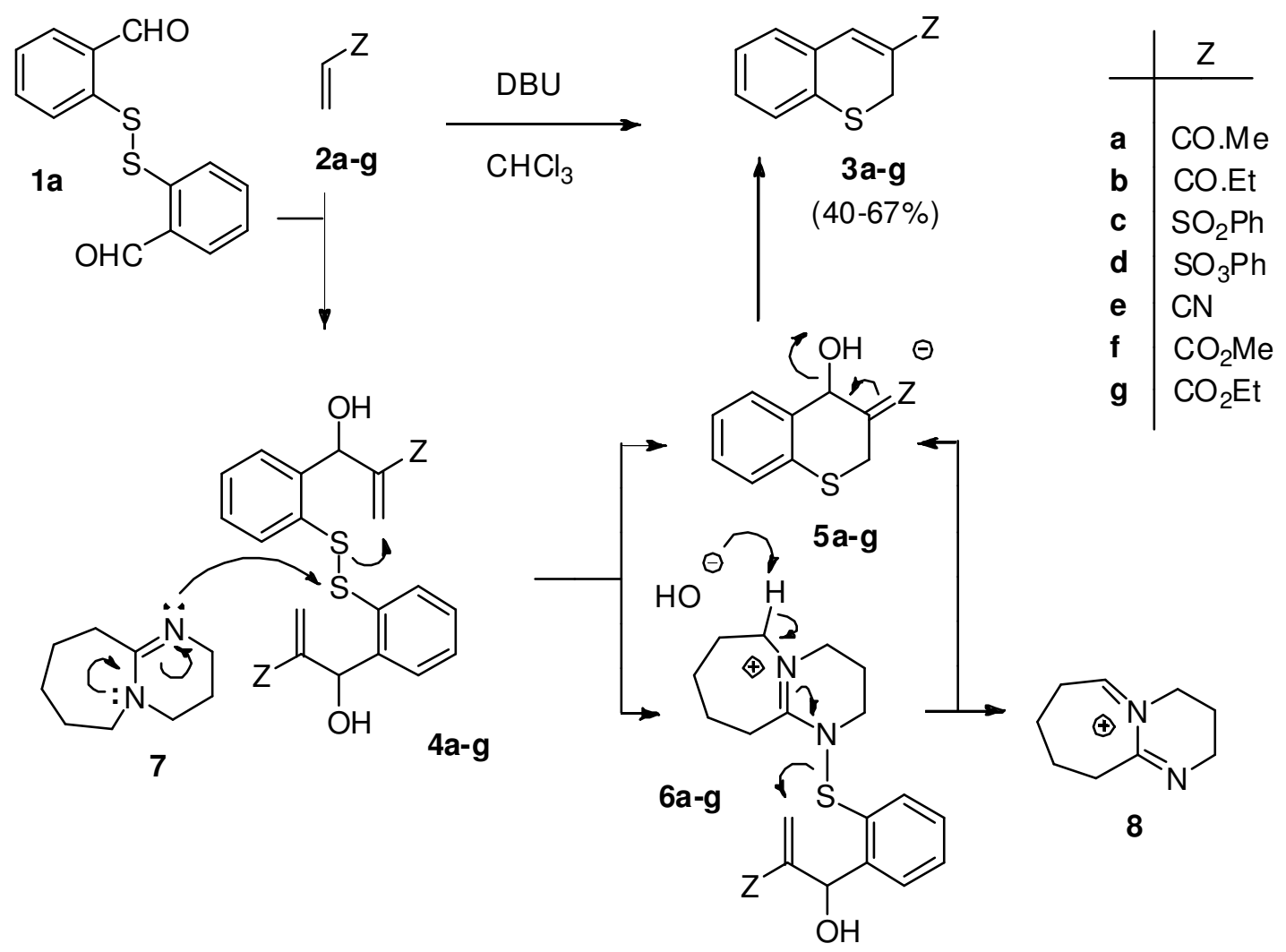

Scheme 1. Synthetic pathway and putative mechanism to account for the formation of the thiochromenes 3a-g. ${ }^{6}$

\section{Results and Discussion}

In order to investigate the potential of DBU to serve as a disulfide reducing agent, solutions of the nine disulfides 1a-i (Scheme 2) in chloroform were treated with DBU in the same molar ratios and under the same reaction conditions used in the previously reported Baylis-Hillman reactions, ${ }^{6}$ but without any activated alkene. [Under these conditions, accommodation of the nucleofugal sulfide by a pre-formed Baylis-Hillman adduct (as suggested in Scheme 1) would be precluded.] In most cases, the expected mercaptans (9a-f) were, in fact, isolated in low to moderate yield (13 - 53\%), demonstrating the ability of DBU to effect reductive cleavage of the disulfide bonds in these compounds. The carboxylic acid derivatives 1g-i, however, afforded crystalline products, NMR analysis of which initially suggested possible trapping of the putative oxidised DBU cation 8 (Scheme 1). Single-crystal X-ray analysis (Figure 1) of the product obtained using the dicarboxylic acid $\mathbf{1} \mathbf{i}$, however, confirmed the formation of the corresponding mono-DBU-disulfide salt 10i. ${ }^{11}$ Formation of the salts $10 \mathrm{~g}-\mathbf{i}$ prompted synthesis of the corresponding methyl esters $\mathbf{1 d - f}$. 
When the substrate 1a was dissolved in $\mathrm{CDCl}_{3}$ alone, no change was observed after 14 days, confirming that DBU is, in fact, responsible for the observed disulfide cleavage. When the disufide 1a was treated with DBU in the dark, normal disulfide cleavage was observed thus excluding a photo-induced, free-radical process.
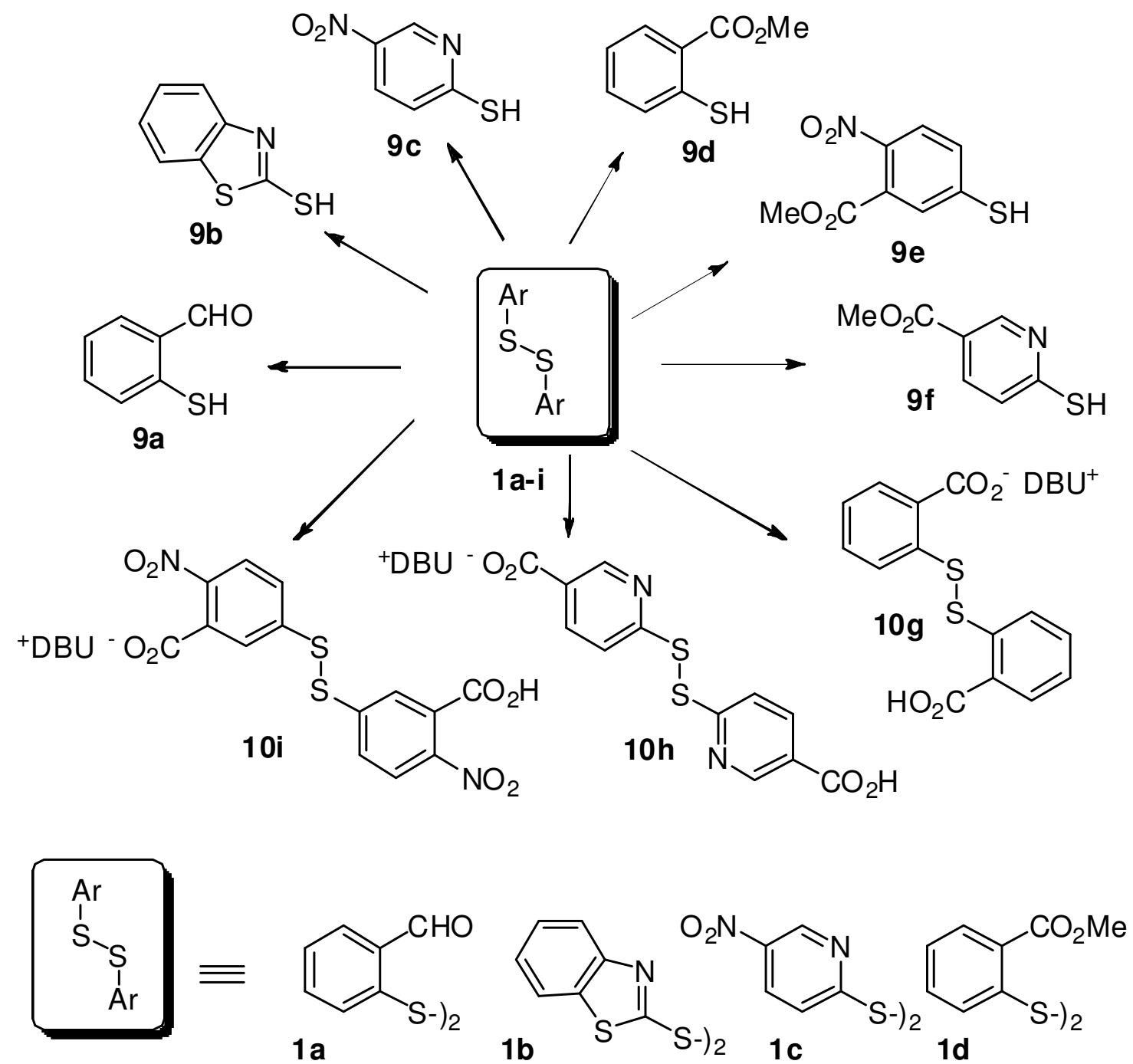<smiles>COC(=O)c1cc(S)ccc1[N+](=O)[O-]</smiles>

$1 e$<smiles>COC(OC)c1ccc(S)nc1</smiles>

$1 f$<smiles>O=C(O)c1ccccc1S</smiles>

19<smiles>O=C(O)c1ccc(S)nc1</smiles>

$1 \mathrm{~h}$<smiles>O=C(O)c1cc(S)ccc1[N+](=O)[O-]</smiles>

$1 \mathrm{i}$

Scheme 2. Reaction of disulfides 1a-i with DBU in chloroform. 


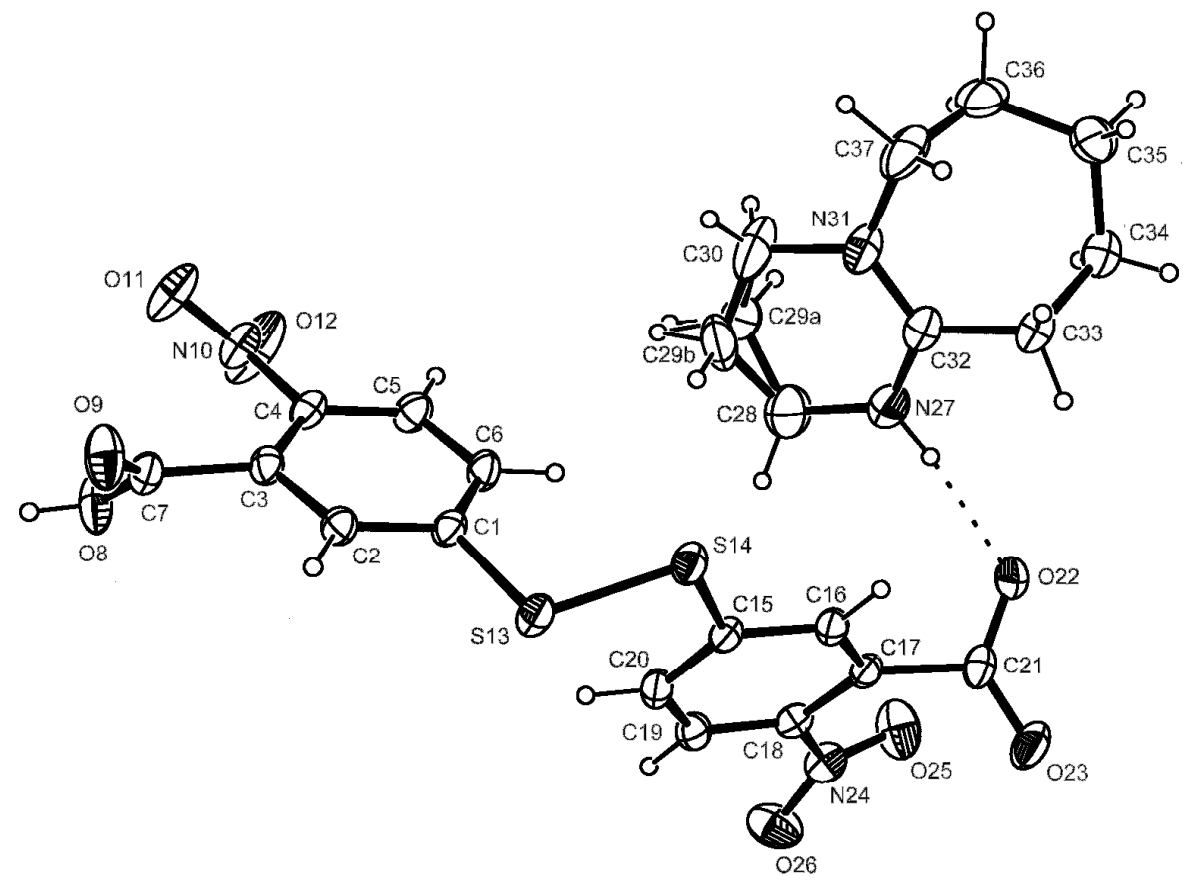

Figure 1. X-Ray crystal structure of the mono-DBU salt 10i of dicarboxylic acid 1i showing the crystallographic numbering for the asymmetric unit and thermal ellipsoids drawn at the $50 \%$ probability level.

\section{Conclusions}

DBU is clearly capable of direct reductive cleavage of the diaryl and heterodiaryl disulfides 1a-f. Optimization and extension of the methodology to aliphatic disulfides may provide an effective alternative, in certain applications, to the use of more established reagent systems. The thiophilicity of DBU 7 in these reactions may be attributable to the intramolecular delocalisation effects illustrated in structure 7 in Scheme 1.

\section{Experimental Section}

General. Reagents, as supplied by Aldrich-Sigma, and solvents were used without further purification. ${ }^{1} \mathrm{H}$ and ${ }^{13} \mathrm{C}$ NMR spectra were recorded on Bruker AMX400 or Avance $\mathrm{II}^{+} 600$ $\mathrm{MHz}$ spectrometers, and were calibrated using solvent signals; coupling constants are given in Hertz (Hz). Melting points were determined using a hot-stage apparatus, and are uncorrected. IR spectra were recorded on a Perkin Elmer Spectrum 100 FT-IR spectrometer. High-resolution mass spectra were recorded by the University of Stellenbosch Mass Spectrometry Unit. Flash chromatography was carried out using Merck silica gel 60 [230-240 mesh (particle size 0.040- 
$0.063 \mathrm{~mm})$ ] and preparative layer chromatography was conducted using silica gel $60 \mathrm{PF}_{254}$. HPLC was carried out on a Partisil 10 Magnum 6 normal phase column using a Spectra-Physics P100 isocratic pump and a Waters K1410 differential refractometry detector.

General procedures and analytical data for new compounds are as follows.

\section{Reactions of DBU with disulfides (1a-f)}

General procedure, exemplified by the preparation of 2-mercaptobenzaldehyde (9a)

DBU $(0.11 \mathrm{~mL}, 0.75 \mathrm{mmol})$ was added slowly to a stirred solution of 2,2'-dithiodibenzaldehyde $(0.1 \mathrm{~g})$ in $\mathrm{CHCl}_{3}(0.7 \mathrm{~mL})$. The mixture was further stirred in a stoppered flask for 2 weeks. Flash chromatography [elution with hexane-EtOAc (1:1)] gave the known compound, ${ }^{12}$ 2mercaptobenzaldehyde 9a $(0.15 \mathrm{~g}, 49 \%)$ as a yellow oil, HPLC of which afforded analytical material (Found M-H: 137.0055. $\mathrm{C}_{7} \mathrm{H}_{5} \mathrm{OS}$ requires: 137.0061); $v_{\max } / \mathrm{cm}^{-1}$ (neat) $2612(\mathrm{~S}-\mathrm{H})$ and $1686(\mathrm{C}=\mathrm{O}) ; \delta_{\mathrm{H}}\left(400 \mathrm{MHz} ; \mathrm{CDCl}_{3}\right) 5.96(1 \mathrm{H}, \mathrm{d}, J=3.49 \mathrm{~Hz}, \mathrm{SH}), 7.41(1 \mathrm{H}, \mathrm{t}, J=7.45 \mathrm{~Hz}, 5-$ H), $7.58(1 \mathrm{H}, \mathrm{dd}, J=7.48$ and $1.37 \mathrm{~Hz}, 6-\mathrm{H}), 7.85(1 \mathrm{H}, \mathrm{t}, J=8.08 \mathrm{~Hz}, 4-\mathrm{H}), 7.93(1 \mathrm{H}, \mathrm{d}, J=$ $7.97 \mathrm{~Hz}, 3-\mathrm{H})$ and $10.14(1 \mathrm{H}, \mathrm{s}, \mathrm{CHO}) ; \delta_{\mathrm{C}}\left(100 \mathrm{MHz} ; \mathrm{CDCl}_{3}\right) 127.4(\mathrm{C}-5), 128.2(\mathrm{C}-4), 129.3$ (C-6), 130.4 (C-3), 134.1 (C-1), 134.8 (C-2) and 192.1 (CHO); m/z 137 (M+100\%).

Other known compounds to be isolated were:

2-Mercapto-1,3-benzothiazole (9b) as a yellow crystalline solid $(0.08 \mathrm{~g}, 53 \%)$, m.p. $154-156{ }^{\circ} \mathrm{C}$ (Lit. ${ }^{13} 177-179{ }^{\circ} \mathrm{C}$ ) (Found $\mathrm{M}^{+}: 65.9779 . \mathrm{C}_{7} \mathrm{H}_{4} \mathrm{NS}_{2}$ requires: 165.9785); ); $v_{\max } / \mathrm{cm}^{-1}$ (neat) 2572 $(\mathrm{S}-\mathrm{H})$ and $1464(\mathrm{C}=\mathrm{N}) ; \delta_{\mathrm{H}}\left(400 \mathrm{MHz} ; \mathrm{CDCl}_{3}\right) 3.52(1 \mathrm{H}, \mathrm{s}, \mathrm{SH}), 7.28(1 \mathrm{H}, \mathrm{m}, 5-\mathrm{H}), 7.37-7.39$ $\left(2 \mathrm{H}, \mathrm{m}, 4 \mathrm{H}\right.$ and 7-H ), $7.46(1 \mathrm{H}, \mathrm{m}, 6-\mathrm{H}) ; \delta_{\mathrm{C}}\left(150 \mathrm{MHz} ; \mathrm{CDCl}_{3}\right) 112.4(\mathrm{C}-5), 121.8(\mathrm{C}-6), 125.1$ (C-4), 127.6 (C-7), 130.5 (C-3a), 140.5 (C-7a), 191.2 (C-2); m/z $166\left(\mathrm{M}^{+}, 100 \%\right)$.

Methyl 2-mercaptobenzoate (9d) ${ }^{14}$ as a yellow oil $(0.03 \mathrm{~g}, 20 \%)$ (Found $\mathrm{M}^{+}: 167.0167$. $\mathrm{C}_{8} \mathrm{H}_{7} \mathrm{O}_{2} \mathrm{~S}$ requires: 167.0171); $v_{\max } / \mathrm{cm}^{-1}$ (neat) $2556(\mathrm{~S}-\mathrm{H})$ and $1704(\mathrm{C}=\mathrm{O}) ; \delta_{\mathrm{H}}(400 \mathrm{MHz}$; $\left.\mathrm{CDCl}_{3}\right) 3.84(1 \mathrm{H}, \mathrm{s}, \mathrm{SH}), 3.97\left(3 \mathrm{H}, \mathrm{s}, \mathrm{OCH}_{3}\right), 7.19-7.22(1 \mathrm{H}, \mathrm{m}, \mathrm{Ar}-\mathrm{H}), 7.40(1 \mathrm{H}, \mathrm{t}, J=7.73 \mathrm{~Hz}$, Ar-H), $7.74(1 \mathrm{H}, \mathrm{d}, J=8.17 \mathrm{~Hz}, \mathrm{Ar}-\mathrm{H})$ and $8.05(1 \mathrm{H}, \mathrm{d}, J=7.01 \mathrm{~Hz}, \mathrm{Ar}-\mathrm{H}) ; \delta_{\mathrm{C}}(100 \mathrm{MHz}$; $\left.\mathrm{CDCl}_{3}\right) 52.4\left(\mathrm{OCH}_{3}\right), 125.5,125.9,127.3,131.5,133.1$ and $140.4(\mathrm{Ar}-\mathrm{C})$ and $166.9(\mathrm{C}=\mathrm{O}) ; \mathrm{m} / z$ $167\left(\mathrm{M}^{+}, 100 \%\right)$.

\section{Analytical data for new compounds are as follows}

2-Mercapto-4-Nitropyridine (9c) as a brown solid (0.0582 g, 30\%), m.p. 96-98 ${ }^{\circ} \mathrm{C}$ (Found $\mathrm{M}^{+}$: 154.9900. $\mathrm{C}_{5} \mathrm{H}_{3} \mathrm{~N}_{2} \mathrm{O}_{2} \mathrm{~S}$ requires: 154.9915); $v_{\max } / \mathrm{cm}^{-1}$ (neat) $2511(\mathrm{~S}-\mathrm{H})$ and $1565(\mathrm{C}=\mathrm{N}) ; \delta_{\mathrm{H}}(400$ $\left.\mathrm{MHz} \mathrm{CDCl}_{3}\right) 6.81(1 \mathrm{H}, \mathrm{d}, J=9.63 \mathrm{~Hz}, 6-\mathrm{H}), 8.33(1 \mathrm{H}, \mathrm{dd}, J=9.53$ and $1.99 \mathrm{~Hz}, 5-\mathrm{H})$ and 9.07 $(1 \mathrm{H}, \mathrm{s}, 2-\mathrm{H}) ; \delta_{\mathrm{C}}\left(100 \mathrm{MHz} ; \mathrm{CDCl}_{3}\right) 119.8,132.1,142.7,145.3$ and $165.0(\mathrm{Ar}-\mathrm{C}) ; \mathrm{m} / z 155\left(\mathrm{M}^{+}\right.$, $100 \%)$.

Methyl 2-mercapto-6-nitrobenzoate (9e) as a yellow oil (0.017 g, 44\%) (Found $\mathrm{M}^{+}: 212.0018$. $\mathrm{C}_{8} \mathrm{H}_{6} \mathrm{NO}_{4} \mathrm{~S}$ requires: 212.0022); ); $v_{\max } / \mathrm{cm}^{-1}$ (neat) $2612(\mathrm{~S}-\mathrm{H})$ and $1732(\mathrm{C}=\mathrm{O}) ; \delta_{\mathrm{H}}(400 \mathrm{MHz}$; $\left.\mathrm{CDCl}_{3}\right) 3.72\left(3 \mathrm{H}, \mathrm{s}, \mathrm{OCH}_{3}\right), 5.42(1 \mathrm{H}, \mathrm{m}, \mathrm{SH}), 7.60(2 \mathrm{H}, \mathrm{m}, \mathrm{Ar}-\mathrm{H}), 7.82(1 \mathrm{H}, \mathrm{m}, \mathrm{Ar}-\mathrm{H})$ and 8.19 
$(1 \mathrm{H}, \mathrm{m}, \mathrm{Ar}-\mathrm{H}) ; \delta_{\mathrm{C}}\left(100 \mathrm{MHz} ; \mathrm{CDCl}_{3}\right) 53.3\left(\mathrm{OCH}_{3}\right), 111.1,124.9,126.2,128.3,133.9$ and 146.2 $\left(\right.$ Ar-C) and $171.1(\mathrm{C}=\mathrm{O}) ; \mathrm{m} / \mathrm{z}, 212\left(\mathrm{M}^{+}, 52 \%\right)$ and $259(100 \%)$.

Methyl 6-mercaptopyridine-3-carboxylate (9f) as a yellow oil (0.02 g, 13\%) (Found $\mathrm{M}^{+}$: 170.0276. $\mathrm{C}_{7} \mathrm{H}_{8} \mathrm{NO}_{2} \mathrm{~S}$ requires: 170.0273); ); $v_{\max } / \mathrm{cm}^{-1}$ (neat) $2541(\mathrm{~S}-\mathrm{H})$ and $1714(\mathrm{C}=\mathrm{O}) ; \delta_{\mathrm{H}}$ $\left(400 \mathrm{MHz} ; \mathrm{CDCl}_{3}\right) 3.92(1 \mathrm{H}, \mathrm{s}, \mathrm{SH}), 3.95\left(1 \mathrm{H}, \mathrm{s}, \mathrm{OCH}_{3}\right),, 7.61(1 \mathrm{H}, \mathrm{d}, J=8.32 \mathrm{~Hz}, \mathrm{Ar}-\mathrm{H})$, $8.21(1 \mathrm{H}, \mathrm{dd}, J=8.31$ and $2.24 \mathrm{~Hz}, \mathrm{Ar}-\mathrm{H}), 9.10(1 \mathrm{H}, \mathrm{s}, 2-\mathrm{H}) ; \delta_{\mathrm{C}}\left(100 \mathrm{MHz} ; \mathrm{CDCl}_{3}\right) 52.9$ $\left(\mathrm{OCH}_{3}\right), 125.4,138.20,138.23,151.58$ and $151.60(\mathrm{Ar}-\mathrm{C})$ and $165.7(\mathrm{C}=\mathrm{O}) ; \mathrm{m} / \mathrm{z} 170\left(\mathrm{M}^{+}, 30 \%\right)$ and $182(100 \%)$..

\section{Esterification of disulfide dicarboxylic acids (1g-i)}

General procedure, exemplified by the preparation of methyl 2-(methoxycarbonylphenyl)disulfanylbenzoate (1d)

$\mathrm{H}_{2} \mathrm{SO}_{4}(0.4 \mathrm{~mL})$ was added to $\mathrm{MeOH}(30 \mathrm{~mL})$, followed by 2,2'-dithiodibenzoic acid $1 \mathrm{~g}(6 \mathrm{~g}$, $0.02 \mathrm{~mol})$, and the resulting mixture was refluxed for $5 \mathrm{~h}$. After cooling, $\mathrm{H}_{2} \mathrm{O}(30 \mathrm{~mL})$ was added and the mixture was stirred for several minutes, before adding further $\mathrm{H}_{2} \mathrm{O}(30 \mathrm{~mL})$ followed by satd. aq. $\mathrm{NaHCO}_{3}(15 \mathrm{~mL})$. The precipitated solid was filtered off and washed with a little $\mathrm{H}_{2} \mathrm{O}$ to give methyl 2-(methoxycarbonylphenyl)disulfanylbenzoate $1 \mathbf{d}$ as a cream powder (13.3 g, 49\%), m.p. $172-173{ }^{\circ} \mathrm{C}$ [Found $\left(\mathrm{M}-\mathrm{C}_{2} \mathrm{H}_{7}\right)^{+}$: 303.0676. $\mathrm{C}_{14} \mathrm{H}_{7} \mathrm{O}_{4} \mathrm{~S}_{2}$ requires: 302.97857]; $v_{\max } / \mathrm{cm}^{-}$ ${ }^{1}$ (neat) $1661(\mathrm{C}=\mathrm{O}) ; \delta_{\mathrm{H}}\left(400 \mathrm{MHz} ; \mathrm{CDCl}_{3}\right) 3.98\left(6 \mathrm{H}, \mathrm{s}, \mathrm{OCH}_{3}\right), 7.23(2 \mathrm{H}, \mathrm{t}, J=7.57 \mathrm{~Hz}, \mathrm{Ar}-\mathrm{H})$, $7.40(2 \mathrm{H}, \mathrm{m}, \mathrm{Ar}-\mathrm{H}), 7.52(2 \mathrm{H}, \mathrm{d}, J=8.07 \mathrm{~Hz}, \mathrm{Ar}-\mathrm{H})$ and $8.05(2 \mathrm{H}, \mathrm{dd}, J=7.78$ and $1.22 \mathrm{~Hz}, \mathrm{Ar}-$ $\mathrm{H}) ; \delta_{\mathrm{C}}\left(100 \mathrm{MHz} ; \mathrm{CDCl}_{3}\right) 52.3\left(\mathrm{OCH}_{3}\right), 125.5,125.9,127.3,131.5,133.1$ and $140.4(\mathrm{Ar}-\mathrm{C})$ and $166.9(\mathrm{C}=\mathrm{O})) ; \mathrm{m} / \mathrm{z} 303\left[\left(\mathrm{M}-\mathrm{C}_{2} \mathrm{H}_{7}\right)^{+}, 30 \%\right]$ and $325(100 \%)$.

Methyl 2-(2-methoxycarbonyl-3-nitrophenyl)disulfanyl-6-nitrobenzoate (1e) as a yellow oil (0.03 g, 57\%) (Found $\mathrm{MH}^{+}: 425.0009 . \mathrm{C}_{16} \mathrm{H}_{13} \mathrm{~N}_{2} \mathrm{O}_{8} \mathrm{~S}_{2}$ requires: 425.01077); $v_{\max } / \mathrm{cm}^{-1}$ (neat) 1724 $(\mathrm{C}=\mathrm{O}) ; \delta_{\mathrm{H}}\left(400 \mathrm{MHz} ; \mathrm{CDCl}_{3}\right) 3.92\left(6 \mathrm{H}, \mathrm{s}, \mathrm{OCH}_{3}\right), 7.87-7.94(6 \mathrm{H}$, series of multiplets, Ar-H); $\delta_{\mathrm{C}}\left(100 \mathrm{MHz} ; \mathrm{CDCl}_{3}\right) 53.6\left(\mathrm{OCH}_{3}\right), 125.1,126.8,127.2,128.4,129.0,142.5(\mathrm{Ar}-\mathrm{C})$ and 167.7 $(\mathrm{C}=\mathrm{O})$.

Methyl 6-[(5-methoxycarbonyl-2-pyridyl)disulfanyl]pyridine-3-carboxylate (1f) as a cream solid $(1.7 \mathrm{~g}, 70 \%)$, m.p. $149-151{ }^{\circ} \mathrm{C}$ [Found $\left(\mathrm{M}-\mathrm{C}_{2} \mathrm{H}_{7}\right)^{+}: 305.0597 . \mathrm{C}_{12} \mathrm{H}_{5} \mathrm{~N}_{2} \mathrm{O}_{4} \mathrm{~S}_{2}$ requires: 304.9692]; $v_{\max } / \mathrm{cm}^{-1}$ (neat) $1716(\mathrm{C}=\mathrm{O}) ; \delta_{\mathrm{H}}\left(400 \mathrm{MHz} ; \mathrm{CDCl}_{3}\right) 3.92\left(6 \mathrm{H}, \mathrm{s}, \mathrm{OCH}_{3}\right), 7.64(2 \mathrm{H}, \mathrm{d}$, $J=8.41 \mathrm{~Hz}, \mathrm{Ar}-\mathrm{H}), 8.18(2 \mathrm{H}, \mathrm{d}, J=8.38 \mathrm{~Hz}, \mathrm{Ar}-\mathrm{H})$ and $9.04(2 \mathrm{H}, \mathrm{s}, \mathrm{Ar}-\mathrm{H}) ; \delta_{\mathrm{C}}(100 \mathrm{MHz}$; $\left.\mathrm{CDCl}_{3}\right) 52.6\left(\mathrm{OCH}_{3}\right), 124.1,125.4,138.1,150.5,164.8(\mathrm{Ar}-\mathrm{C})$ and $\left.165.8(\mathrm{C}=\mathrm{O})\right) ; \mathrm{m} / \mathrm{z} 305[(\mathrm{M}$ $\left.\left.-\mathrm{C}_{2} \mathrm{H}_{7}\right)^{+}, 100 \%\right]$

\section{Formation of mono-DBU disulfide dicarboxylic acid salts (10g-i)}

The mono-DBU salt (10i). The general procedure described for the synthesis of 2mercaptobenzaldehyde (9a) was followed using 5,5'-dithiobis-(2-nitrobenzoic acid) (0.1 g, 0.9 $\mathrm{mmol})$, DBU $(0.1 \mathrm{~mL}, 2 \mathrm{mmol})$ and $\mathrm{CHCl}_{3}(1.0 \mathrm{~mL})$. Work up afforded a yellow solid which was recrystallised from EtOH to give the mono-DBU salt $10 \mathbf{i}(0.12 \mathrm{~g}, 68 \%)$ as yellow crystals, m.p. $217-220{ }^{\circ} \mathrm{C} ; v_{\max } / \mathrm{cm}^{-1}$ (neat) $1689(\mathrm{C}=\mathrm{O})$ and $1560(\mathrm{C}=\mathrm{N}) ; \delta_{\mathrm{H}}\left(600 \mathrm{MHz} ; \mathrm{CDCl}_{3}\right) 1.63-1.71$ 
(6H, m, DBU-CH $\left.\mathrm{CH}_{2}\right), 1.89\left(2 \mathrm{H}, \mathrm{m}, \mathrm{DBU}-\mathrm{CH}_{2}\right), 2.65\left(2 \mathrm{H}, \mathrm{m}, \mathrm{DBU}-\mathrm{CH}_{2}\right), 3.22(2 \mathrm{H}, \mathrm{br}$ s, DBU$\left.\mathrm{CH}_{2}\right)$, ca. 3.5 (4H, overlapping $\mathrm{H}_{2} \mathrm{O}$ signal, DBU-CH $\left.\mathrm{CH}_{2}\right), 7.48(1 \mathrm{H}, \mathrm{dd}, J=8.46$ and $2.20 \mathrm{~Hz}$, Ar$\mathrm{H}), 7.69(\mathrm{H}, \mathrm{m}, \mathrm{Ar}-\mathrm{H}), 7.73(1 \mathrm{H}, \mathrm{s}, \mathrm{Ar}-\mathrm{H})$ and $10.15\left(1 \mathrm{H}, \mathrm{s}, \mathrm{CO}_{2} \mathrm{H}\right) ; \delta_{\mathrm{C}}\left(150 \mathrm{MHz} ; \mathrm{CDCl}_{3}\right) 18.9$, 23.4, 25.9, 28.2, 31.5, 37.5, 47.8, 53.3 (DBU-C), $99.5(\mathrm{C}=\mathrm{N}), 124.0,125.5,126.2,139.7,147.0$ and $165.3($ Ar-C) and $168.8(\mathrm{C}=\mathrm{O})$.

The mono-DBU salt $(\mathbf{1 0 g}) \cdot(0.39 \mathrm{~g}, 70 \%)$ as a cream solid, m.p. $213-216{ }^{\circ} \mathrm{C} ; v_{\max } / \mathrm{cm}^{-1}$ (neat) $1672(\mathrm{C}=\mathrm{O})$ and $1572(\mathrm{C}=\mathrm{N}) ; \delta_{\mathrm{H}}\left(600 \mathrm{MHz} ; \mathrm{CDCl}_{3}\right)$ 1.59-1.66 $\left(6 \mathrm{H}, \mathrm{m}, \mathrm{DBU}-\mathrm{CH}_{2}\right), 1.88-1.91$ $\left(2 \mathrm{H}, \mathrm{m}, \mathrm{DBU}-\mathrm{CH}_{2}\right), 2.75\left(2 \mathrm{H}, \mathrm{m}, \mathrm{DBU}-\mathrm{CH}_{2}\right), 3.45-3.55\left(6 \mathrm{H}\right.$, overlapping $\mathrm{H}_{2} \mathrm{O}$ signal, DBU$\left.\mathrm{CH}_{2}\right), 7.06(1 \mathrm{H}, \mathrm{t}, J=7.23 \mathrm{~Hz}, \mathrm{Ar}-\mathrm{H}), 7.13(1 \mathrm{H}, \mathrm{t}, J=7.51 \mathrm{~Hz}, \mathrm{Ar}-\mathrm{H}), 7.45(1 \mathrm{H}, \mathrm{d}, J=8.00 \mathrm{~Hz}$, Ar-H), $7.80(1 \mathrm{H}, \mathrm{d}, J=7.43 \mathrm{~Hz}, \mathrm{Ar}-\mathrm{H})$ and $8.27\left(1 \mathrm{H}, \mathrm{s}, \mathrm{CO}_{2} \mathrm{H}\right) ; \delta_{\mathrm{C}}\left(150 \mathrm{MHz} ; \mathrm{CDCl}_{3}\right) 18.9$, 23.3, 25.9, 28.1, 31.3, 37.5, 47.7, 53.2 (DBU-C), $97.6(\mathrm{C}=\mathrm{N}), 123.6,123.7,128.3,129.9,132.8$ and $165.3(\mathrm{Ar}-\mathrm{C})$ and $168.3\left(\mathrm{CO}_{2} \mathrm{H}\right)$.

The mono-DBU salt (10h) $(0.33 \mathrm{~g}, 59 \%)$ as a brown solid, m.p. $207-209{ }^{\circ} \mathrm{C} ; v_{\max } / \mathrm{cm}^{-1}$ (neat) $1662(\mathrm{C}=\mathrm{O})$ and $1606(\mathrm{C}=\mathrm{N}) ; \delta_{\mathrm{H}}\left(400 \mathrm{MHz} ; \mathrm{CDCl}_{3}\right)$ 1.64-1.72 (6H, m, DBU-CH $)_{2}, 1.98(2 \mathrm{H}, \mathrm{m}$, DBU-CH $\left.{ }_{2}\right), 2.86\left(2 \mathrm{H}, \mathrm{m}, \mathrm{DBU}-\mathrm{CH}_{2}\right), c a .3 .5\left(6 \mathrm{H}\right.$, overlapping $\mathrm{H}_{2} \mathrm{O}$ signal, DBU- $\left.\mathrm{CH}_{2}\right), 7.55$ $(1 \mathrm{H}, \mathrm{d}, J=8.31 \mathrm{~Hz}, \mathrm{Ar}-\mathrm{H}), 8.20(1 \mathrm{H}, \mathrm{dd}, J=8.32$ and $2.03 \mathrm{~Hz}, \mathrm{Ar}-\mathrm{H}), 8.84(1 \mathrm{H}, \mathrm{s}, \mathrm{Ar}-\mathrm{H})$ and $9.04\left(1 \mathrm{H}, \mathrm{s}, \mathrm{CO}_{2} \mathrm{H}\right) ; \delta_{\mathrm{C}}\left(150 \mathrm{MHz} ; \mathrm{CDCl}_{3}\right) 18.9,23.4,26.0,28.3,31.5,37.6,47.8,53.3$ (DBUC), $108.6(\mathrm{C}=\mathrm{N}), 118.4,138.4,150.5,158.2$ and $165.3(\mathrm{Ar}-\mathrm{C})$ and $166.3(\mathrm{C}=\mathrm{O})$.

Crystal data for the mono\{1,8-diazabicylo[5.4.0]undec-7-ene\} salt of 5,5'-dithiobis-(2nitrobenzoic acid)(10i). $\left(\mathrm{C}_{14} \mathrm{H}_{7} \mathrm{~N}_{2} \mathrm{O}_{8} \mathrm{~S}_{2}\right)^{-}\left(\mathrm{C}_{9} \mathrm{H}_{17} \mathrm{~N}_{2}\right)^{+}, M=548.58,0.18 \times 0.16 \times 0.13 \mathrm{~mm}^{3}$, triclinic, space group $P(-1)$ (No. 2), $a=10.1019(8), b=10.4227(8), c=12.3805(9) \AA, \alpha=$ 77.899(2), $\beta=74.919(2), \gamma=80.698(2)^{\circ}, V=1222.73(16) \AA^{3}, Z=2, D_{\mathrm{c}}=1.490 \mathrm{~g} / \mathrm{cm}^{3}, F_{000}=$ 572 , MoK $\alpha$ radiation, $\lambda=0.71073 \AA, T=173(2) \mathrm{K}, 2 \theta_{\max }=56.7^{\circ}, 48890$ reflections collected, 6080 unique $\left(\mathrm{R}_{\mathrm{int}}=0.0435\right)$. Final $\mathrm{GooF}=1.049, R_{l}=0.0361, w R_{2}=0.0912, R$ indices based on 5006 reflections with $I>2 \sigma(I)$ (refinement on $F^{2}$ ), 353 parameters, 2 restraints. Lp and absorption corrections applied, $\mu=0.275 \mathrm{~mm}^{-1}$. Primary dihedral angles in the disulfide ion include C1-S13-S14-C15 -90.40(7) ${ }^{\circ}$, S13-S14-C15-C20 15.1(1) ${ }^{\circ}$ and S14-S13-C1-C6 21.0(1) ${ }^{\circ}$. One of the methylene groups (C29) is statistically disordered over two positions (a, b). Crystallographic data (excluding structure factors) for the structure reported in this paper have been deposited with the Cambridge Crystallographic Data Centre as supplementary publication no. CCDC-816182. Copies of the data can be obtained free of charge on application to CCDC, 12 Union Road, Cambridge CB21EZ, UK (fax:(44) 1223-336-033; e-mail: deposit@ccdc.cam.ac.uk).

\section{Acknowledgements}

The authors thank the National Research Foundation (NRF: GUN 2069255) and Rhodes University for a bursary (D.N), Rhodes University, the University of Cape Town and the NRF for generous financial support. Any opinion, findings and conclusions or recommendations 
expressed in this material are those of the authors and therefore the NRF does not accept any liability in regard thereto.

\section{References and Notes}

1. Ingall, A. H. In Comprehensive Heterocyclic Chemistry, Boulton, A. J.; McKillop, A. Eds., Vol. 3, Pergamon Press: Oxford, 1984, p 934.

2. Tercio, J.; Ferreira, B.; Catani, V.; Comasseto, J. V. Synthesis 1987, 149-153.

3. Arnoldi, A.; Carughi, M. Synthesis 1988, 155-157.

4. Kobayashi, K.; Konishi, H.; Kitamura, T.; Morikawa, O.; Nakahashi, R. J. Chem. Soc., Perkin Trans. 1 1999, 1547-1552.

5. (a) Bode, M. L.; Kaye, P. T. J. Chem. Soc., Perkin Trans. 1 1990, 2612-2613. (b) Bode, M.

L.; Kaye, P.T.J. Chem. Soc., Perkin Trans. 1 1993, 1809-1811. (c) Familoni, O. B.; Kaye, P. T.; Klaas, P. J. Chem. Commun. 1998, 2563-2564. (d) Kaye, P. T.; Nocanda, X. W. J. Chem. Soc., Perkin Trans. 1 2000, 1331-1332.

6. Kaye, P. T.; Nocanda, X. W. Synthesis 2001, 2389-2392.

7. (a) Humphrey, R. E.; Hawkins, J. M. Anal. Chem., 1964, 36 (9), 1812-1814. (b) Dmitrenko, O.; Thorpe, C.; Bach, R. D. J. Org. Chem. 2007, 77(22), 8298-8307.

8. Changyuan, L.; Bucher, G.; Sander, W. ChemPhysChem. 2004, 5, 399-402.

9. Taniguchi, T.; Fujii, T.; Idota, A.; Ishibashi, H. Org. Lett., 2009, 11 (15), 3298-3301.

10. Inoue, H.; Tamura, S. Chem. Commun. 1986, 858-859.

11. The cation and anion are linked by the H-bond $\mathrm{N} 27-\mathrm{H} \cdots \mathrm{O} 22$ with $\mathrm{N} \cdots \mathrm{O} 2.823(2) \AA$; the anions are linked in infinite chains via a strong head-to-tail $\mathrm{H}$-bond $\mathrm{O} 8-\mathrm{H} \cdots \mathrm{O} 22^{\mathrm{i}}$ with $\mathrm{O} \cdots \mathrm{O}$ $2.473(2) \AA(\mathrm{i}=x,-1+y, 1+z)$. The molecular symmetry indicated by the NMR spectra in some cases is attributed to dynamic proton transfer in solution.

12. Gallagher, T.; Pardoe, D. A.; Porter, R. A. Tetrahedron Lett. 2000, 41, 5415-5418.

13. Benati, L.; Montevecchi, P. C. J. Org. Chem. 1981, 46, 4570-4573.

14. Katz, L.; Karger, L. S.; Schroeder, W.; Cohen, M. S. J. Org. Chem. 1953, 18, 1380-1402. 